\title{
Temperature-Dependence of Deposition Rate and Current Efficiency in Platinum Electrodeposition at a Fixed Average Current Density
}

\author{
M. Saitou*, S. Teruya and S. M. Asadul Hossain
}

Department of Mechanical Systems Engineering, University of the Ryukyus, 1 Senbaru Nishihara-cho Okinawa, Japan, 903-0213

\begin{abstract}
We have investigated the formation of a platinum thin film from a simple solution of dihydrogen hexachloroplatinate in a low temperature range of 283 to $295 \mathrm{~K}$ by a pulse current technique. Despite the low temperature, the platinum thin film observed with a confocal laser scanning microscope does not comprise particle aggregates but dense layer with a small surface roughness that tends to saturate at an initial growth stage. The deposition rate and current efficiency at a fixed average current density are evidently dependent on temperature. The analysis of the mol weight of platinum deposit based on two electrochemical reactions proposed in platinum electrodeposition elucidates the temperature dependence characterized by a thermal activation process.
\end{abstract}

Keywords: Deposition rate, current efficiency, platinum, pulse current electrodeposition.

\section{INTRODUCTION}

Platinum materials have a large number of applications to jewelry, electronic components, electrodes, thermal sensors, catalysis, and magnetic materials. Owing to the high cost of bulk platinum materials, they are replaced with Pt thin films. Therefore, electrodeposition of the Pt thin films is so low in cost that many kinds of electrolytes [1-3], additives [4-6], substrates for platinum electrodeposition [7-9], and fabricating processes have been proposed.

For instance, in platinum black that comprises platinum particle aggregates and has a porous structure, the effect of temperature [1] and additives [10-14] on the size of particle was investigated. No platinum particle aggregates at an initial growth stage developed into a dense thin film with a small surface roughness. If diffusion of adatoms reduced from metal ions on a substrate is limited, the deposited film has a porous structure as known by calculations based on a ballistic growth model and diffusion-limited aggregate model [15].

The reduction process of a platinum atom from a Pt(IV) ion analyzed by cyclic voltammetry was proposed as the following equation $[13,16]$ :

$P t(I V)+4 e^{-} \rightarrow P t$.

Another reduction process studied by a chronopotentionmetric technique was given by [17]:

$P t(I V)+P t+e^{-} \rightarrow P t+P t(I I I)$,

which indicates catalysis by the deposited platinum. However, there have been very few studies on the temperature-dependence of the deposition rate and current efficiency

\footnotetext{
*Address correspondece to this author at the Department of Mechanical Systems Engineering, University of the Ryukyus, 1 Senbaru Nishihara-cho Okinawa, Japan, 903-0213; Tel: +81-98-895-8635; Fax: +81-98-895-8636; E-mail: saitou@tec.u-ryukyu.ac.jp
}

in platinum electrodeposition on the basis of the two electrochemical reactions.

In this study, a pulse current and low temperature electrodeposition technique, which is known to lessen the size of grain in a thin film [12-14], will be shown to allow the formation of the dense platinum film from a solution of dihydrogen hexachloroplatinate free of additives.

The purposes of the present paper show that the pulse current and low temperature electrodeposition technique from the solution of dihydrogen hexachloroplatinate free of additives allows the dense layer growth with a smooth surface, and that the temperature dependence of the deposition rate and current efficiency at a fixed average current density is well explained by the analysis of the mol weight of platinum deposit based on the two reduction processes of platinum ion including the catalysis process.

\section{EXPERIMENTAL SETUP}

Experiments were performed using a solution consisting of the only one component $\left(\mathrm{molL}^{-1}\right): \mathrm{H}_{2} \mathrm{PtCl}_{6} \cdot 6 \mathrm{H}_{2} \mathrm{O}, 0.1$. The concentration was much higher than that in other studies $[7-9,12-14]$ because of the lower electrical resistance of the solution and a smaller change in the concentration of platinum ions during electrodeposition.

A poly-crystalline nickel substrate of $4 \times 30 \mathrm{~mm}^{2}$ and carbon plate of $10 \times 30 \mathrm{~mm}^{2}$ were prepared for a cathode and anode electrode. The nickel substrate appears to be a mirror in appearance and has a standard deviation of surface height $0.1 \pm 0.02 \mu \mathrm{m}$, which was measured with a confocal laser scanning microscope (Keyence VF7500) to an accuracy of $0.01 \mu \mathrm{m}$ in height. As far as we know, no electroless deposition of platinum on the nickel electrode in a solution of $\mathrm{H}_{2} \mathrm{PtCl}_{6}$ occurs [18]. After the two electrodes were cleaned by a wet process, the one side of the nickel electrode was electrically isolated by using an insulating tape. The two electrodes were located parallel in the quiescent solution in the 
temperature range of 283 to $295 \mathrm{~K}$, which was much lower than the conventional temperature range of 343 to $363 \mathrm{~K}$.

A square wave pulse current having an amplitude of 5 $\mathrm{mA}$, current on-time of $5 \mathrm{~ms}$, and current off-time of $5 \mathrm{~ms}$ was provided with a power supply (Advantest, R6145). All the experiments were performed at an average pulse current density of $6.25 \mathrm{mAcm}^{-2}$. After pulse current electrodeposition, the nickel electrode was fully rinsed in distilled water and dried. The weight of the platinum thin film was measured with an electric balance (A\&D HR-60) to an accuracy of $0.1 \mathrm{mg}$.

The surface morphology of the platinum thin film was observed with the confocal laser scanning microscope, which provided a digitized microscope image with a resolution of $780 \times 564$ pixels. The surface roughness of the platinum thin film was estimated at the standard deviation $\mathrm{w}(\mathrm{t})$ :

$w(t)=\left(\frac{1}{N} \sum_{i=1}^{N}\left(h_{i}-\bar{h}\right)^{2}\right)^{1 / 2}$,

where $t$ is the growth time, $\mathrm{N}$ is the number of pixel, $\mathrm{h}_{\mathrm{i}}$ is the height of the deposit at a position $\mathrm{i}$ and $\bar{h}$ is the average height formed by $\mathrm{N}$ points.

\section{EXPERIMENTAL RESULTS AND DISCUSSION}

We here assume that the two electrochemical reactions in Eqs. (1) and (2) occur during platinum electrodeposition. When the Pt electrodeposition obeys only Eq. (1), the deposit weight at the fixed current density does not change with temperature. If both Eqs. (1) and (2) simultaneously occur in $\mathrm{Pt}$ electrodeposition, the temperature-dependent deposit weight at the fixed pulse current density will be well explained. As the catalysis process in Eq. (2) is dependent on temperature, the ratio of the current used for the reduction of $\mathrm{Pt}(\mathrm{IV})$ to the fixed current changes with temperature.

The combination of Eqs. (1) and (2) yields

$(x+y) P t(I V)+y P t+(4 x+y) e^{-} \rightarrow x P t+y P t(I I I)+y P t$,

where $\mathrm{x}$ and $\mathrm{y}$ are the mole number of Pt(IV) that is involved with the electrochemical reactions in Eqs. (1) and (2). Equating the number of moles of product expressed in electrochemical terms with the number of moles of product expressed as molecular weight, we have from Eq. (4),

$\frac{y}{x}=\frac{m_{p}}{\frac{m}{t}} \cdot \frac{I}{F}-4$,

where $m_{p}$ is the mole weight of platinum, $m$ is the weight of the deposited platinum, $t$ is the deposition time, $I$ is the current, and $\mathrm{F}$ is the Faraday constant. Eq. (5) indicates that the mole ratio $y / x$ can be determined by the deposition rate $\mathrm{m} / \mathrm{t}$ and current I.

\subsection{Microscopic Images of the Dense Platinum thin Film}

Microscopic images of the platinum thin film electrodeposited at a total current on-time of $600 \mathrm{~s}$ for three kinds of temperature are shown in Figs. (1a, b and $\mathbf{c})$. The total ontime is defined by the summation of the current on-time during electrodeposition. The microscopic image in Fig. (1a), which was electrodeposited at $283 \mathrm{~K}$, shows several granular mounds on the surface, however, it is apparent that the plati- num thin films are not particle aggregates but dense layers. At the higher temperature, the granular mounds almost disappear as shown in Figs. (1b and $\mathbf{c})$.

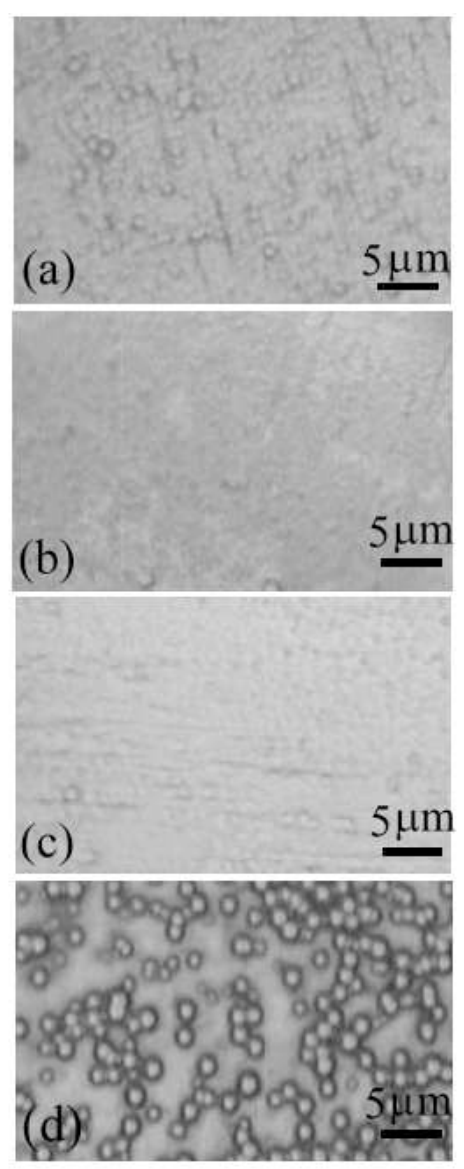

Fig. (1). Images of the platinum electrodeposits observed with the confocal laser scanning microscope. The platinum thin films were grown for a total on-time of 600 s at a temperature of (a) $283 \mathrm{~K}$, (b) $288 \mathrm{~K}$, and (c) $295 \mathrm{~K}$. The platinum particle aggregates in Fig. (1d) were electrodeposited for $300 \mathrm{~s}$ at $331 \mathrm{~K}$ by a DC current technique.

According to the equivalent circuit model [19], the potential is almost applied to the electric double layer described by resistance and capacitance. In pulse current electrodeposition, the potential is not as large as that in direct current electrodeposition because the capacitance plays a role of bypass condenser at a higher frequency.

On the other hand, as the number of atoms arriving at the cathode electrode in pulse electric deposition is much larger than that in direct current electrodeposition, the growth velocity of thin film in a short period of time (during the current on-time) in pulse current electrodeposition becomes larger than that in direct electric electrodeposition. As the dynamic scaling theory [15] predicts, the number of atoms does not affect surface growth mechanisms. However, the correlation length $\xi$ is affected by the growth rate $\mathrm{v}, \xi \sim v^{-\theta}$ where $\theta$ is the positive exponent. The correlation length is related to the grain size $\mathrm{d}, \xi \sim d$. Hence, the grain size in pulse current electrodeposition becomes smaller. In addition, the correlation length in phase transition is related to tem- 
perature $\mathrm{T}, \xi \sim \exp \left(B /\left(T_{R}-T\right)^{1 / 2}\right)$ where $\mathrm{B}$ is the constant and $\mathrm{T}_{\mathrm{R}}$ is the roughness transition temperature. As the correlation length at a lower temperature becomes shorter, the grain size at the lower temperature becomes smaller. Hence, the pulse current at low temperature yields thin films with small grain size.

The reduction process of $\mathrm{Pt}(\mathrm{IV})$ comprising three steps has been studied with cyclic voltammetry and reported [10, 20]. The three steps such as $\mathrm{PtCl}_{6}^{-2}+2 e^{-} \rightarrow \mathrm{PtCl}_{4}^{-2}+2 \mathrm{Cl}^{-}$, $\mathrm{PtCl}_{6}^{-2}+4 e^{-} \rightarrow \mathrm{Pt}+6 \mathrm{Cl}^{-}$and $\mathrm{PtCl}_{4}^{-2}+2 e^{-} \rightarrow \mathrm{Pt}+4 \mathrm{Cl}^{-}$are related to the plateau and peak in the cyclic volammograms. At a higher cathode potential, the adsorption of $\mathrm{H}$ atoms on a formed $\mathrm{Pt}$ island prior to the reduction of $\mathrm{H}^{+}$to $\mathrm{H}_{2}$ gas is observed.

In our experiment, no evolution of hydrogen is observed owing to the lower cathode potential. This supports no trace of hydrogen evolution left on the film surface such as spherically-shaped pits in Fig. (1). Hence, the current will be almost used to synthesize the platinum film.

As a reference, the platinum particle aggregates grown at $331 \mathrm{~K}$ for $300 \mathrm{~s}$ by a D.C. (direct current) electrodeposition technique is shown in Fig. (1d). Denuded areas in which no platinum particle aggregation occurs are observed. Hence, D.C. and high temperature electrodeposition enhances the formation and growth of platinum particle aggregates. The surface morphology in Fig. (1d) at a later growth time becomes a thin film comprising granular islands of micrometer order. The surface appears very rough in comparison with those in Fig. (1a, b and c).

\subsection{Effect of Temperature on the Deposition Rate}

In Fig. (2), the weight of the platinum thin film linearly increases with the total on-time. If the platinum electrodeposition obeys only Eq. (1), the deposit weight at the fixed average current density is independent of temperature because the current used for the formation of platinum keeps constant. However, as shown in Fig. (2), the deposit weight of the platinum thin film electrodeposited at the fixed average current density of $6.25 \mathrm{~mA} \mathrm{~cm}^{-2}$ (the average current I, 5 $\mathrm{mA}$ ) increases with temperature.

The straight line best fitted to the data in Fig. (2) yields the deposition rate, $\mathrm{m} / \mathrm{t}$ in Eq. (5). In Fig. (3), the deposition rate and current efficiency increase with temperature. In addition, the current efficiency at $295 \mathrm{~K}$ is about 1.5 times higher than that at $283 \mathrm{~K}$. The temperature dependence of the current efficiency is so large that it cannot be explained by the evolution of hydrogen.

If we take into consideration not only Eq. (1) but also Eq. (2), Eq. (5) will give a solution for the temperature dependence of the deposition rate and current efficiency. In such a case, the current efficiency is calculated by

$1-\frac{y}{4 x+y}=\frac{4}{4+\frac{y}{x}}$.

The second term on the left hand side in Eq. (6) describes the fraction of the current used in Eq. (2). Using Eqs. (5) and (6), plots of the ratio $y / x$ and current efficiency are shown in

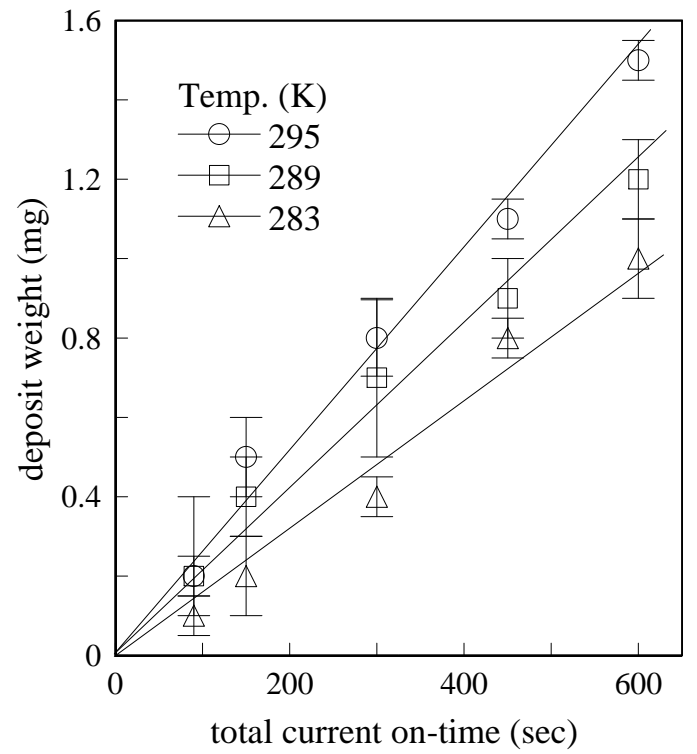

Fig. (2). A plot of the deposit weight vs. total current on-time for three kinds of temperature. The straight lines best fitted to data indicate the deposition rates.

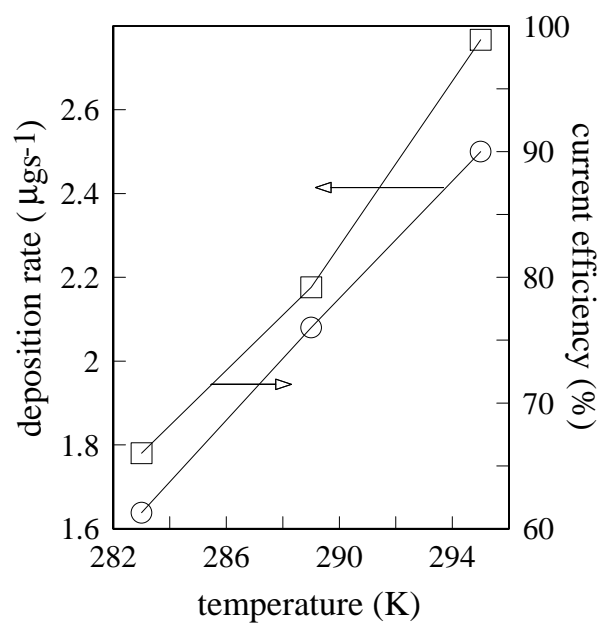

Fig. (3). Temperature dependence of the deposition rate and current efficiency obtained from Fig. (2).

Fig. (4). The current efficiency dependent on temperature, which is due to the presence of Eq. (2), is in good agreement with that in Fig. (3).

Furthermore, plotting the data of $\mathrm{x} / \mathrm{y}$ and $1 / \mathrm{T}$ in a semi$\log$ scale, we make an attempt to elucidate a thermal activation process of platinum electrodeposition. The straight line best fitted to the data in Fig. (5) is expressed by

$\frac{x}{y} \propto \exp \left(-\frac{\Delta G}{k_{B} T}\right)$,

where $k_{B}$ is the Boltzmann's constant, $\Delta G=\Delta G_{1}-\Delta G_{2}$ is the net activation energy, and $\Delta \mathrm{G}_{1}$ and $\Delta \mathrm{G}_{2}$ are the thermal activation energy for the electrochemical reactions in Eq. (1) and Eq. (2). The thermal activation energy given by the slope in Fig. (5) becomes $\Delta \mathrm{G}=2 \pm 0.05$ eV/atom. Hence, $\Delta \mathrm{G}_{1}>\Delta \mathrm{G}_{2}$ and the large thermal activation energy support a large change in the weight of the platinum electrodeposit with temperature. 


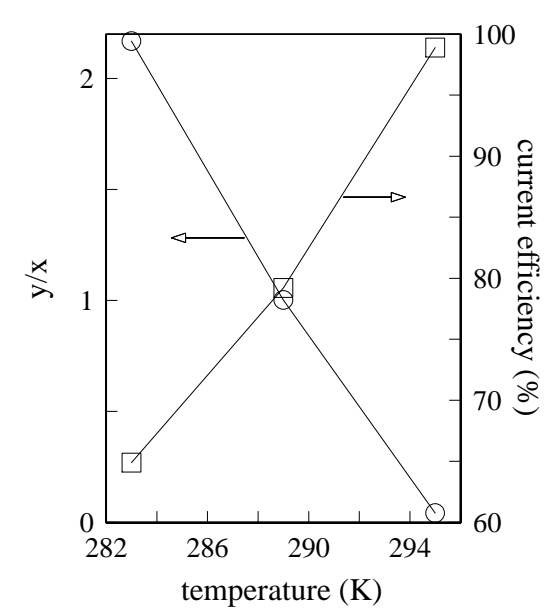

Fig. (4). Deposition rate and current efficiency calculated from Eqs. (5) and (6).

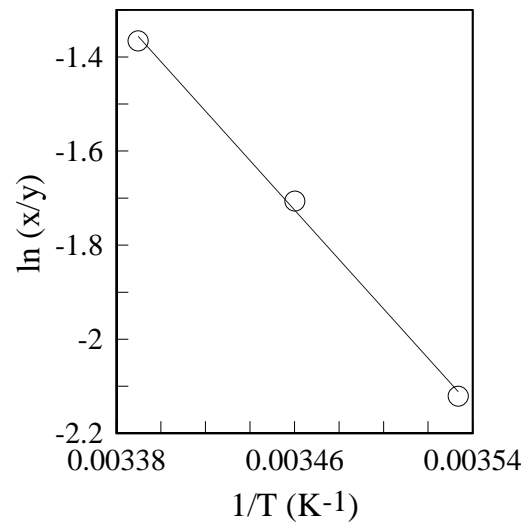

Fig. (5). A semi-logarithmic plot of $\mathrm{x} / \mathrm{y}$ vs. reciprocal temperature. The slope best fitted to the data yields the activation en$\operatorname{ergy} \Delta \mathrm{G}=2 \pm 0.05 \mathrm{eV}$.

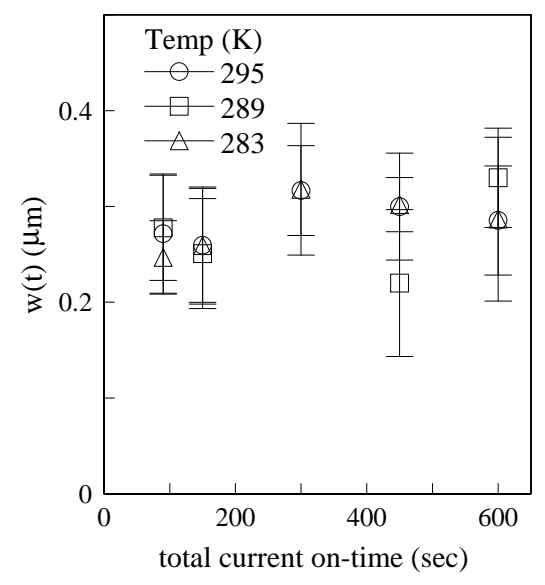

Fig. (6). Dependence of the standard deviation of surface height on the total current-on time for a variety of temperature. The standard deviation of the platinum thin film of $0.78 \mu \mathrm{m}$ in film thickness was calculated from the surface height measured with the confocal laser scanning microscope.

\subsection{Effect of Temperature on the Surface Roughness}

The standard deviation of surface roughness in Fig. (6) shows that $w(t)$ keeps constant about $0.3 \mu \mathrm{m}$ irrespective of the total current on-time and temperature. In general, w(t) for deposited films increases with time and over a crossover time tends to saturate $[15,17]$. As the saturated value 0.28 $\mu \mathrm{m}$ on average is larger than the standard deviation $0.1 \mu \mathrm{m}$ of surface roughness of the nickel substrate, the standard deviation of the platinum thin film is found to increase to 0.3 $\mu \mathrm{m}$ at $100 \mathrm{~s}$. The standard deviation of surface roughness, which is independent of temperature in this study, suggests that surface diffusion at low temperature has an insignificant effect on the thermal-activated surface growth.

Thus, the dense platinum thin film with the smooth surface is produced by the pulse current technique at low temperature from the solution free of additives.

\section{CONCLUSIONS}

The platinum thin films that are dense and smooth in surface appearance are produced from the aqueous solution of dihydrogen hexachloroplatinate in the low temperature range of 283 to $297 \mathrm{~K}$. The effect of temperature on the deposit weight and current efficiency is well explained by the mol ratio $y / x$ derived from the combination of the two electrochemical reactions proposed in platinum electrodeposition. The standard deviation of surface roughness shows that it tends to saturate at the initial growth stage and the saturated standard deviation is independent of time and temperature.

\section{REFERENCES}

[1] Pushpavanam, M. High speed platinum deposition from a sulphamate formulation. J. Appl. Electrochem., 2006, 36, 10691074.

[2] Thompson, S. D.; Jordan, L. R.; Shukla, A. K.; Forsyth, M. Platinum electrodeposition from $\mathrm{H}_{3} \mathrm{Pt}\left(\mathrm{SO}_{3}\right)_{2} \mathrm{OH}$ solutions. $J$. Electroanal. Chem., 2001, 515, 61-70.

[3] Obert, J.; Lalvani, S. B. Development of a bath for codeposition of copper and platinum. J. Appl. Electrochem., 2004, 34, 397-401.

[4] Sieben, J. M.; Duarte, M. N. E.; Mayer, C. E.; Bazãn, J. C. Influence of ethylene glycol, ethanol and formic acid on platinum and ruthenium electrodeposition on carbon support material. J. Appl. Electrochem., 2009, 39, 1045-1051.

[5] Chen, F.; Ye, L.; Li, J.; Li, J.; Wang, X. Shape-controlled fabrication of platinum electrocatalyst by pulse electrodeposition. Electrochem. Commun., 2008, 10, 476-479.

[6] G-Peña, O. I.; Chapman, T. W.; Vong, Y. M.; A-López, R. Study of adsorption of citrate on Pt by CV and EQCM. Electrochim. Acta., 2008, 53, 5549-5554.

[7] Thompson, S. D.; Jordan, L. R.; Forsyth, M. Platinum electrodeposition for polymer electrolyte membrane fuel cells. Electrochim. Acta., 2001, 46, 1657-1663.

[8] Stoychev, D.; Papoutsis, A.; Kelaidopulou, A.; Kokkinidis, G.; Milchev, A. Electrodeposition of platinum on metallic and nonmetallic substrate. Mater. Chem. Phys., 2001, 72, 360-365.

[9] Hosseini, M. G.; Sajjadi, S. A. S.; Momeni, M. M. Electrodeposition of platinum metal on titanium and anodised titanium from P salt: application to electro-oxidation of glycerol. Surf. Eng., 2007, 23, 419-424.

[10] Saitou, M. Electrochemical characterization of platinum black electrodeposited from electrolyte including lead acetate trihydrate. Surf. Coating Technol., 2006, 201, 3611-3614.

[11] Duarte, M. M. E. ; Pilla, A. S.; Sieben, J. M.; Mayer, C. E. Platinum particles electrodeposition on carbon substrates. Electrochem. Commun., 2006, 8, 159-164.

[12] Kelaidopoulou, A.; Kokkinidis, G.; Milchev, A. Nucleation and growth of metal catalysts. Part I. electrodeposition of platinum on tungsten. J. Electroanal. Chem., 1998, 444, 195-201.

[13] Chen, S.; Kucernak, A. Electrodeposition of platinum on nanometer-sized carbon electrodes. J. Phys. Chem. B., 2003, 107, 83928402 . 
[14] Nieto, F. J. R.; Pasquale, M. A.; Cabrera, C. R.; Arvia, A. J. Morphology of platinum electrodeposits in the three-dimensional sublayer to full layer range produced under different potential modulations on highly oriented pyrolytic graphite. Langmuir., 2006, 22, 10472-10482.

[15] Barabási, A. L.; Stanley, H. E. Fractal Concepts in Surface Growth.; Cambridge University Press: Cambridge, 1995.

[16] Kim, C. S.; Oh, S. M. Enzyme sensors prepared by electrodeposition on platinized platinum electrodes. Electrochim. Acta., 1996, 41, 2433-2439.
[17] Lingane, J. J. Chronopotentiometric study of the redox characteristics of $\mathrm{PtCl}_{6}{ }^{2-}$ and $\mathrm{PtCl}_{4}{ }^{2-}$ at a platinum electrode. J. Electroanal. Chem., 1964, 7, 94-101.

[18] Schlesinger, M. Paunovic M. Modern Electrodeposition, John Wiley \& Sons: New York, 2000.

[19] Bard, A. J.; Faulkner, L. R. Electrochemical Methods, John Wiley \& Sons: New York, 1980.

[20] Lu, G; Zangari, G. Electrodeposition of platinum on highly oriented pyrolytic graphite. J. Phys. Chem. B., 2005, 109, 79988007.

(C) Saitou et al.; Licensee Bentham Open.

This is an open access article licensed under the terms of the Creative Commons Attribution Non-Commercial License (http://creativecommons.org/licenses/ by-nc/3.0/) which permits unrestricted, non-commercial use, distribution and reproduction in any medium, provided the work is properly cited. 